\title{
Virologen als (Super)Heroic Scientists
}

Erschienen in: Sozialfiguren der Corona-Pandemie Von: Martin Butler, Michael Fuchs, Sina Farzin

Als in Deutschland gerade durch den ersten Lockdown den meisten klar geworden war, dass die Pandemie ernst genommen werden muss, machte eine kleine vierteilige Bildcollage in den sozialen Medien Hoffnung: alles nicht so schlimm, Rettung naht - und zwar in Person eines Wissenschaftlers mit beeindruckender Vita. In der Collage waren drei Screenshots aus verschiedenen Filmen versammelt, in denen der schwarzgelockte Schauspieler Jeff Goldblum als fiktiver Wissenschaftler erfolgreich die Menschheit vor Gefahren durch Saurier, Körperfresser und Aliens zu schützen suchte. Das letzte der vier Bilder zeigte den Virologen Christian Drosten, zum damaligen Zeitpunkt gerade als Corona-Experte durch einen täglichen Podcast in den Aufmerksamkeitsbereich der Öffentlichkeit gerückt - und mit Jeff Goldblum eher lose durch eine augenscheinliche Vorliebe für ungekämmtes Haupthaar verbunden. Über die Textunterschriften wurde eine Assoziationskette hergestellt, die Goldblums wiederholte Darstellung fiktiver heldenhafter Wissenschaftlerfiguren mit Drostens neuer öffentlicher Rolle als CoronaErklärer verband und suggerierte, es handele sich um ein und dieselbe Figur: „Er hat schon gegen Saurier, Körperfresser und Aliens gekämpft. Da vertraue ich inm jetzt auch mit dem Virus." Und um die Einheit der fiktionalen, von Goldblum verkörperten Figuren mit dem Berliner Professor für Virologie zu betonen, wurde letzterem kurzerhand noch eine schwarzrandige Brille aufmontiert, die jenen Modellen ähnelt, die die GoldblumCharaktere in den Blockbustern Jurassic Park (1993) und Independence Day (1996) tragen.

Die Überblendung des Wissenschaftlers Drosten mit fiktiven Wissenschaftlerfiguren aus populären Filmen beruht auf einem scheinbar oberflächlichen, humorgetriebenen Spiel von popkulturellen und alltagsweltlichen Referenzen, die typisch für digitale Memes sind. ${ }^{2}$ Vorausgesetzt wird eine gewisse Vertrautheit mit den aufgerufenen Filmplots, die zugleich gemeinschaftsstiftend wirkt, da sie auf einen geteilten kulturellen Sinnhorizont verweist. Versteht man das Meme, darf man mitlachen, bleiben die Anspielungen nicht als solche nachvollziehbar, gehört man nicht dazu. ${ }^{3}$ Dabei wirkt in der Aneignung und Bearbeitung der Pandemie auf Social-Media-Kanälen vor allem eine Sozialfigur als Knotenpunkt verschiedener kultureller Skripte, die in einer Situation großer kollektiver Verunsicherung Deutungsangebote bieten: der heroic scientist. Als tradiertes Stereotyp der kulturellen Darstellung von Wissenschaftlern bietet der heldenhafte und in dieser Eigenschaft zumeist männliche Forscher als Figur einen Bezugsrahmen, in den die virologischen und epidemiologischen Corona-Experten eingefügt werden können. 
Dabei bilden Wissenschaftler-Stereotype nicht einfach individuelle Subjekte ab oder porträtieren Personen mit Anspruch auf Wahrhaftigkeit, sondern bündeln soziale Erwartungen, Hoffnungen und Ängste, die an die Wissenschaft adressiert werden, in Form einer schematischen

Personalisierung. Gleichzeitig sind sie eingebunden in kulturelle Skripte, d. h. in wiederkehrende Erzählungen, in deren Plots innen bestimmte Funktionen zukommen. Roslynn Haynes zeigt in ihrer Forschung zur Darstellung von Wissenschaftlern in der Literaturgeschichte, dass der heldenhafte Wissenschaftler eines der populärsten und am häufigsten variierten Stereotype in literarischen Wissenschaftserzählungen ist. ${ }^{4}$ Dabei erscheint er in zwei Varianten: als weiser Anführer und als Retter. Erstere Variante verbindet wissenschaftliche Kompetenz mit Klugheit und moralischer Überlegenheit, ein Topos, der seit Francis Bacons Nova Atlantis über H. G. Wells Science-Fiction-Utopien bis in gegenwärtige Erzählungen reicht. Die Figur des Retters ersetzt das spirituelle Versprechen auf Erlösung durch die Aussicht auf Ordnung mittels Logik und Rationalität in einer ansonsten unkontrollierbaren Welt. Beide Varianten finden sich nicht nur in zahllosen Romanen, sondern auch in Filmen, Serien, Erzählungen, Comics und anderen weit verbreiteten Erzählungen, in denen Wissenschaft eine Rolle spielt.

Vor dem Hintergrund dieser Deutungsmuster betreten mit der Pandemie etliche Virolog*innen, Epidemiolog*innen, Mediziner*innen und Public Health Expert*innen die öffentliche Bühne und werden auch durch die Linse dieser kulturellen Prägung beobachtet und interpretiert. Sie sprechen über die Medien als Expert*innen, vermitteln hochspezialisiertes Wissen über das neue Virus und erlangen häufig in kürzester Zeit enorme Bekanntheit, erscheinen sie doch in einer zunehmend unsicheren und bedrohlichen Situation als Aufklärer, die nicht nur die unsichtbare Gefahr benennen, sondern zugleich einordnen, Handlungswissen zur Verfügung stellen und politische Entscheidungsträger*innen beraten. Dabei treten sie auch als Vermittler*innen komplexen und teilweise vorläufigen und unvollständigen Wissens öffentlich in Erscheinung - eine Ausgangskonstellation, die einiges an Spannungspotential birgt.

Als pandemische Sozialfiguren werden Corona-Wissenschaftler jedoch nicht nur als Experten, Aufklärer und Vermittler sichtbar (gemacht), sondern auch als heldenhafte Figuren. Ihre öffentliche Karriere im Verlauf der Pandemie vollzieht sich entlang von Trajektorien, die auf einer vor allem durch das Stereotyp des heroic scientist gerahmten Erwartungshaltung gegenüber wissenschaftlicher Kompetenz beruht. Dies erklärt auch, dass trotz zahlreicher hochkompetenter weiblicher Expertinnen vor allem männliche Wissenschaftler wie Christian Drosten oder Anthony Fauci zu ,Kultfiguren“ werden. So 
werden in der Collage der Jeff Goldblum-Screenshots jene Rollen gewählt, die auf genau diesem Stereotyp beruhen. Die ebenfalls popkulturell bedeutsame Darstellung Goldbums im David Cronenberg Film The Fly wird hingegen ausgespart: sie beruht auf dem Gegenbild des heldenhaften Wissenschaftlers, dem mad scientist, der jenseits aller moralischen und wissenschaftsethischen Grenzen agiert. Dabei prägen auch diese Gegenstereotype die öffentliche Darstellung des Corona-Wissenschaftlers, indem über eingeschliffene Plotmuster Antagonisten zu den heldenhaften Figuren aufgebaut werden. Der Spiegel beispielsweise überschrieb einen Artikel über den Virologen Hendrik Streeck, der in einigen Fragen andere wissenschaftliche Einschätzungen zum Umgang mit der Pandemie als Christian Drosten vertrat mit dem Titel „Der Anti-Held“ (Der Spiegel 44/2020) und raunte seitenlang über das Verhältnis der beiden Wissenschaftler, anstatt die wissenschaftliche Begründung der abweichenden Einschätzungen zu erläutern. ${ }^{5}$

Am schnellsten finden die Verbindungen (pop)kultureller Skripte mit den CoronaWissenschaftlern auf den verschiedenen Social-Media-Plattformen statt, auf denen auch jenseits der Pandemie die eng getaktete und kurzweilige popkulturelle Verbindung von potentiell allem mit allem zelebriert wird. In geteilten Bildern und Verweisen werden aus Experten heroic scientists, die in einem teilweise ironisierenden Verweisspiel als fiktive Superhelden und reale Hoffnungsträger adressiert werden. Über die Reichweite und mit der Geschwindigkeit der virtuellen Plattformen verbreiten sich dabei auch singuläre Werke und Objekte, wie etwa ein Graffiti aus Pegnitz, das Drosten als Superman abbildet, oder eine Gebetskerze, die den amerikanischen Immunologen Anthony Fauci als Heiligenfigur zeigt.

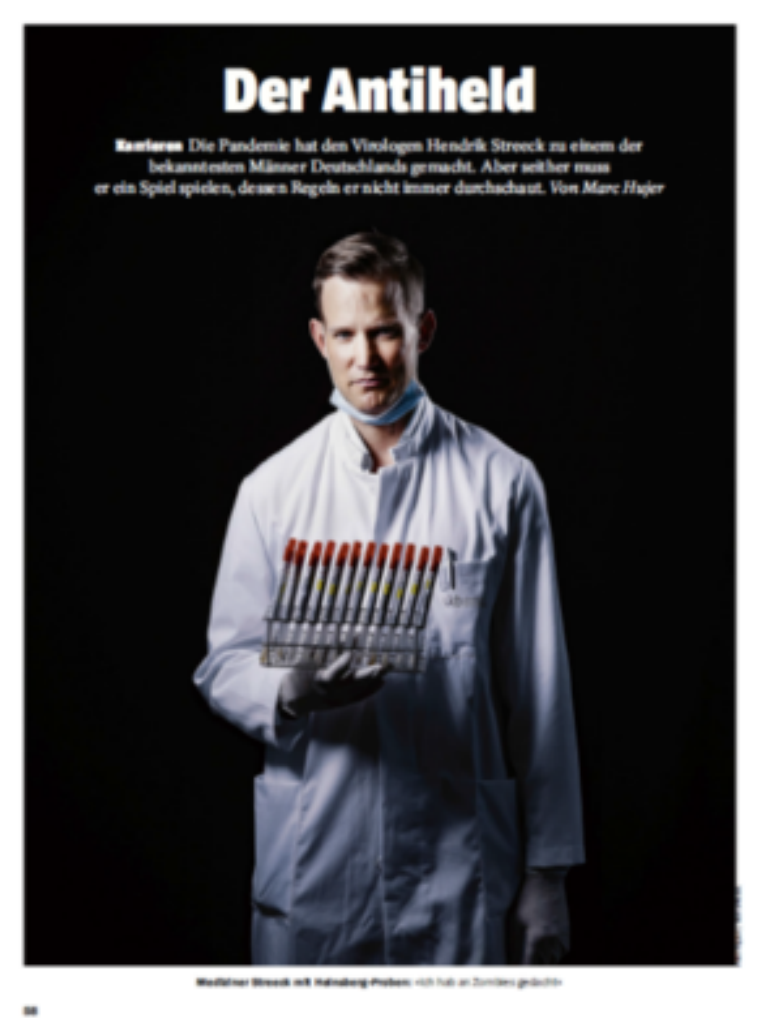

Abb. 2: Virologe Streeck ${ }^{6}$ 


\section{Found today in a street in Lauf an der Pegnitz.} \#StreetArt \#COVID19 \#Drosten

\section{$\leftarrow$ Tweet}

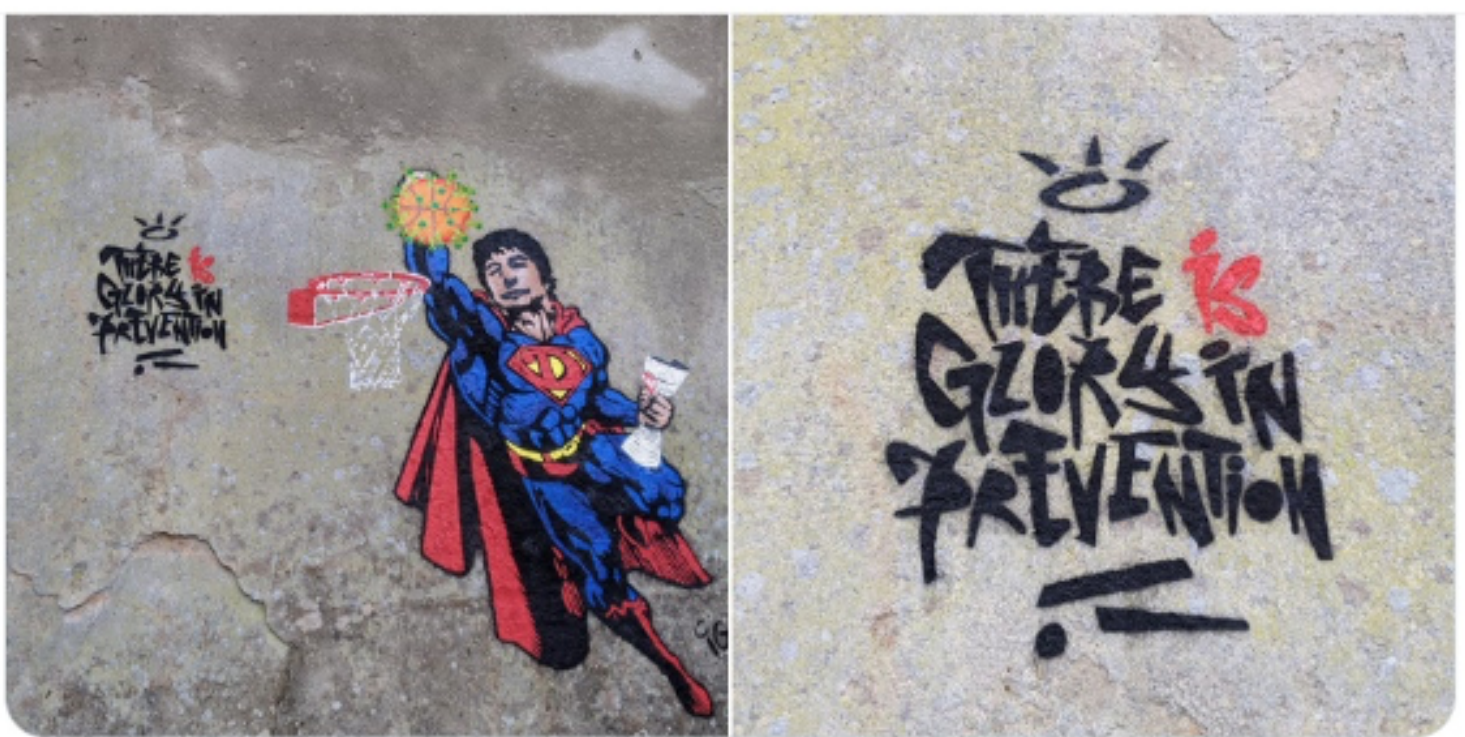

12:57 AM · Aug 30, $2020 \cdot$ Twitter Web App

Abb. 3: Tweet zu Drosten Street Art $^{7}$
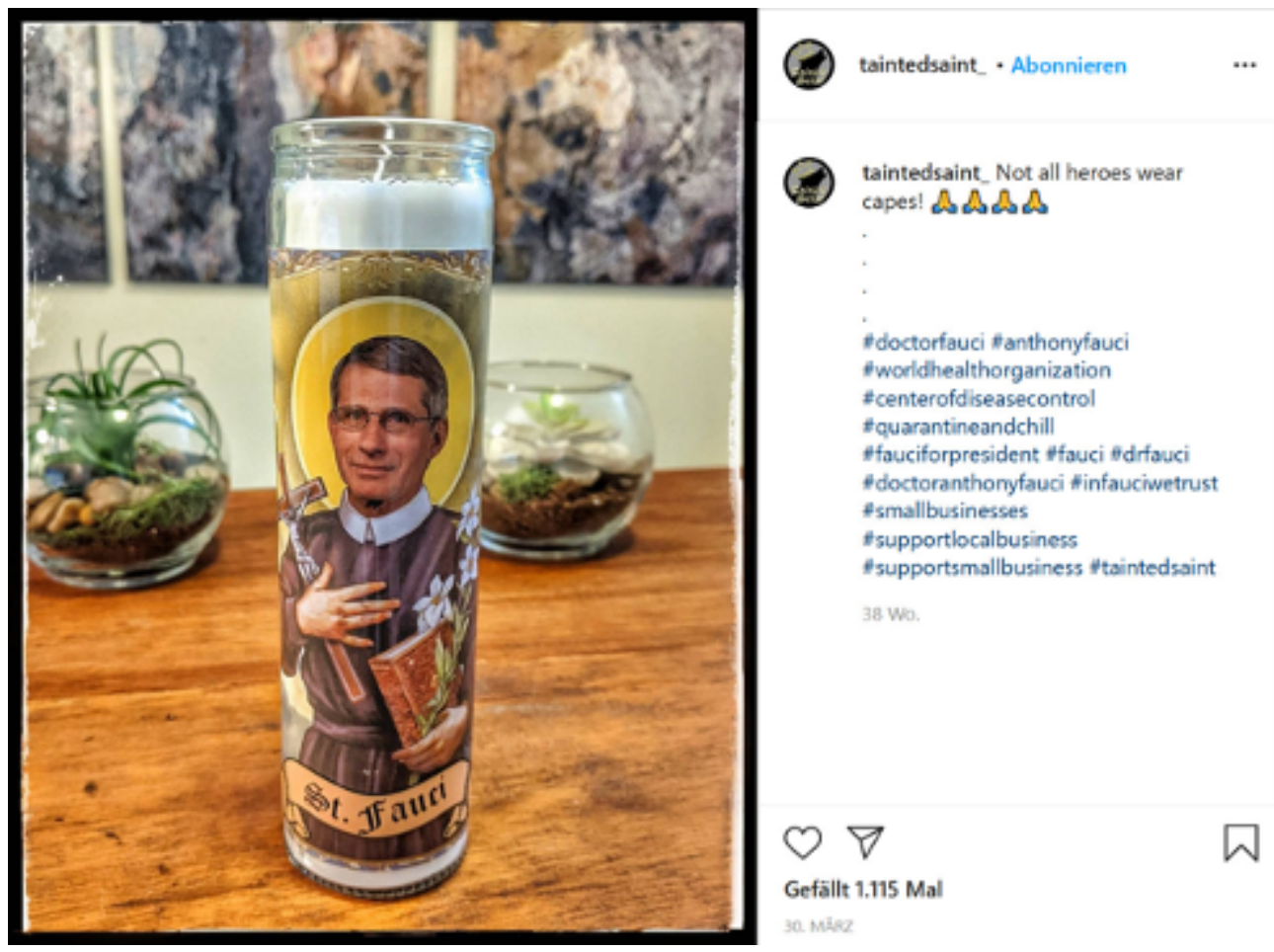

Abb. 4: Gebetskerze mit Fauci-Konterfei auf dem Instagram-Account des OnlineHandels taintedsaint ${ }^{8}$ 
Dabei werden beide Dimensionen des Stereotyps aufgerufen und in unterschiedliche Richtungen ironisierend verstärkt: Als Führungsfiguren werden die heroischen Wissenschaftler neben die etablierten popkulturellen Superhelden gerückt und mit deren übermenschlichen Fähigkeiten verbunden. Artikuliert wird so auch die Hoffnung auf einen Akteur, der durch sein so exzeptionelles wie individuelles Handeln den Schlüssel zur Lösung der Krise in der Hand hält. Das hat sehr wenig mit den tatsächlichen Arbeitsweisen und Handlungsspielräumen der adressierten Wissenschaftler zu tun und sehr viel mit den kulturellen Stereotypen und Erzählungen, die in der akuten Krisensituation aktiviert werden. Und obwohl diese Rückgriffe zumeist durch ironische Aufladung und Überzeichnung gekennzeichnet sind, artikulieren sie doch eine Hoffnung auf die Handlungsfähigkeit der einzelnen Person. Als quasi-religiöse Ikonen wiederum werden die Wissenschaftler als moralisch und ethisch herausgehobene Individuen inszeniert und zu Gegenspielern nicht nur des generellen Pandemiegeschehens, sondern jeglicher dunklen Mächte verklärt. Beide Stränge liefen in einem Tweet der Twitter-Nutzerin Ida Funkhouser, der im Juli 2020 über 14tausend „likes“ erhielt, zusammen. Während der Sommerpause des Podcast, den Christian Drosten gemeinsam mit dem NDR zur Corona-Thematik sendet, wurde angesichts niedriger Fallzahlen in einigen Bundesländern über die Abschaffung der Maskenpflicht diskutiert. Dazu schrieb Ida Funkhouser auf Twitter: „Drosten wie lange hast du noch Urlaub die wollen Maskenpflicht abschaffen" und hing in einem zweiten Tweet ein Meme mit dem Text „Mein Gott, mein Gott, warum hast du mich verlassen?“ an.

Die Verbindung der Hoffnung auf die direkte Intervention in eine politische Debatte durch den Wissenschaftler und seine Adressierung als gottgleiche Instanz verknüpfte beide Stränge des Stereotyps und erhielt unmittelbar eine starke Resonanz.

Derartige Adressierungen zielen allerdings nicht auf eine konsequente Sakralisierung oder religiöse Aufladung ab. Den Urheber*innen und Rezipient*innen dieser und anderer Memes ist in der überwiegenden Mehrheit klar, dass hier eine im Kern aufrichtige Sorge mit den Mitteln des Humors bearbeitet und artikuliert wird. Die Ikonifizierung der Wissenschaftler zitiert und variiert religiöse Motive oberflächlich, ohne die Funktion und

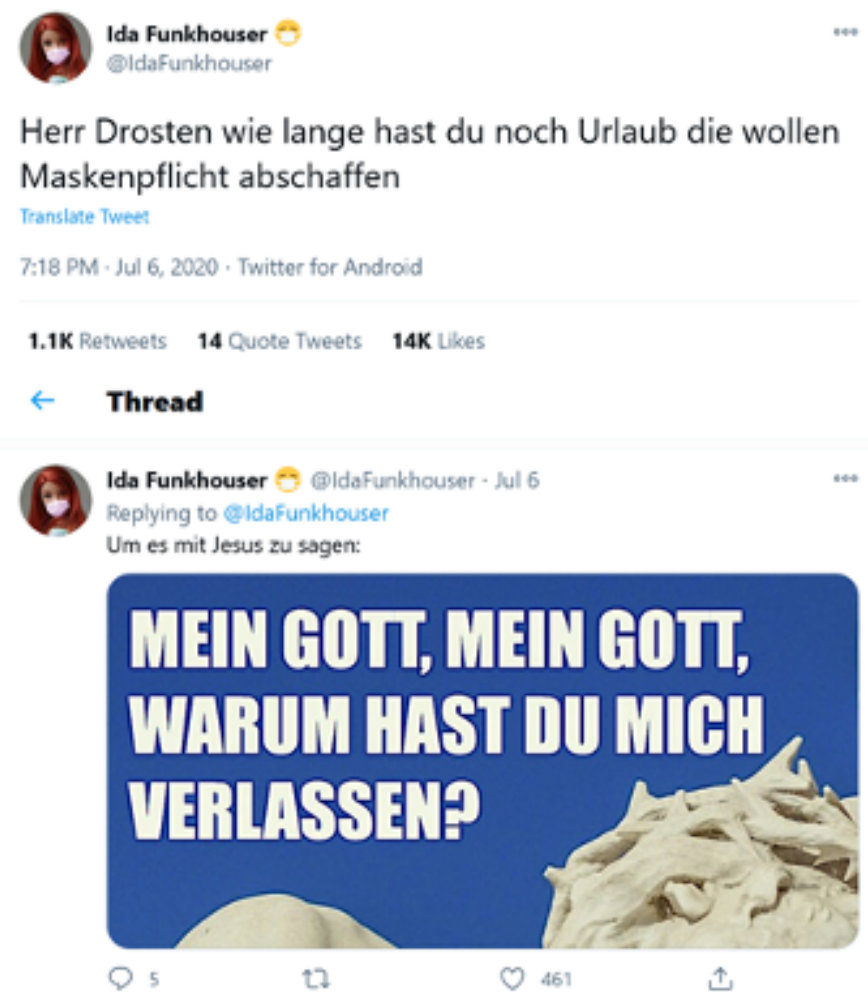

Abb. 5: Tweet von @IdaFunkhouser ${ }^{9}$ Stabilität tatsächlicher religiöser Praktiken der Verehrung zu beanspruchen oder zu benötigen. Es ist daher, so könnte man sagen, gerade dieses Spiel mit den Bruchstellen zwischen wissenschaftlichen und religiösen Geltungsansprüchen, flüchtigen und fest 
etablierten sozialen Praktiken der Anbetung, unterstellter und tatsächlicher politischer Entscheidungskompetenz und die Kurzschlüsse zwischen diesen Ebenen, die den Erfolg dieser Form der Netzkommunikation begründen. Die Heroisierung der Wissenschaftler in den sozialen Medien hat - häufiger als in den herkömmlichen Medien, die sich derselben Stereotype bedienen - etwas dezidiert Flüchtiges, Unernstes, Spielerisches. Religiöse Versatzstücke und Superheldenkräfte werden zitiert und in popkulturell erprobte Praktiken fanförmiger Begeisterung für die Personen eingebunden. Diese rücken nun als charismatische Persönlichkeiten, und eben nicht nur als wissenschaftliche Experten, in den Mittelpunkt der Aufmerksamkeit. Auf einmal ist nicht mehr nur ihre Expertise und Kompetenz von Interesse, sondern ihr Musikgeschmack, ihr Gehalt oder ihr Privatleben. Die Parallelen zu eher star- oder celebrityhaften Formen der Vereinnahmung beschränken sich nicht allein auf eine Publikumshaltung, die der von Fans entspricht (\#teamdrosten), sondern schleusen die heroic scientists als Figuren in die typischen Verwertungskreisläufe der Popkultur ein. Kaffeetassen oder Alltagsmasken mit den Konterfeis von Drosten oder Fauci werden in zahlreichen Variationen angeboten, Twitter-Zitate von Drosten auf T-Shirts und Sticker gedruckt. Dabei erlaubt es die Individualisierung von Produktionsformen in relativ kurzer Zeit, kleine Serien von Merchandising zu entwickeln und so fast in Echtzeit auf aktuelle Debatten, Äußerungen und Konflikte zu reagieren. Angetrieben werden diese Prozesse dabei auch durch die Wissenschaftler selbst, die einerseits immer wieder Befremden und Distanz gegenüber dieser Art der Vereinnahmung äußern, jedoch teilweise auch gezielt Allianzen eingehen. So freute sich Christian Drosten via Twitter über ein ihm gewidmetes Lied der Berliner Punkband ZSK, während Anthony Fauci sich bei Brad Pitt bedankte, der inn in einem Saturday Night Live-Sketch verkörperte, nachdem Fauci in einem Interview eher scherzhaft angemerkt hatte, er würde in einer Verfilmung des Pandemiegeschehens am liebsten von Pitt dargestellt werden.

Diese losen Verbindungen zwischen der traditionsreichen Sozialfigur des heroic scientist, durch Social-Media vermittelter Wissenschaftskommunikation und popkulturellen Praktiken haben die Kommunikationsprozesse um die Pandemie herum schneller, unvorhersehbarer, aber auch lustiger und schärfer gemacht, als es die traditionelle Medienöffentlichkeit bisher gewohnt war. Die momentan im Zentrum dieser Öffentlichkeit stehenden Wissenschaftler*innen werden nach der Pandemie vermutlich größtenteils wieder eher unbeobachtet ihrer Arbeit nachgehen. Was bleiben wird, ist die Figur des heroic scientist, dann vielleicht wieder besetzt mit Jeff Goldblum in einem Science-Fiction-Film, bei dem die Liebhaber*innen realistischer europäischer Autor*innenfilme im dokumentarischen Stil nur abschätzig befinden können: „Die Effekte waren ja ganz nett, aber die Story etwas weit hergeholt."

\section{References}

1. Quelle: https://www.facebook.com/Cineplex.LM/photos/jeff-drosten-goldblum-tothe-rescue-hier-noch-ein-paar-jeff-goldblum-fakten-mit-/3108227249268938/ 
2. Vgl. Shifman, Limor (2014): Memes in Digital Culture. Boston: MIT Press, S. $67 f f$. https://doi.org/10.7551/mitpress/9429.001.0001

3. Vgl. Zappavigna, Michele (2012): Discourse of Twitter and Social Media: How We Use Language to Create Affiliation on the Web. London/New York: Bloomsbury, S. 101

4. Vgl. Haynes, Roslynn. (2003): From Alchemy to Artificial Intelligence: Stereotypes of the Scientist in Western Literature. Public Understanding of Science 12, S. 243-253, S. 245f. https://doi.org/10.1177/0963662503123003

5. Diese stark auf einzelne Wissenschaftler zugespitzte Debatte machen sich auch insbesondere radikale Kritiker zu eigen. Corona-Leugner richten ihre öffentlichen Angriffe auf einzelne Personen (wie etwa Drosten in Deutschland, Anthony Fauci in den USA), um sie, angereichert durch eine verschwörungstheoretische Rhetorik, nicht selten als willige Handlanger politischer Umtriebe zu diffamieren.

6. Quelle: Der SPIEGEL 44/2020, S. 58

7. Quelle: https://twitter.com/4n4Le4/status/1299843662696808450

8. Quelle: https://www.instagram.com/p/B-XZNCop7tV/? utm_source=ig_web_copy_link

9. Quelle: https://twitter.com/ldaFunkhouser/status/1280189386123104257

SUGGESTED CITATION: Butler, Michael; Farzin, Sina; Fuchs, Michael: Virologen als (Super)Heroic Scientists, in: KWI-BLOG, [https://blog.kulturwissenschaften.de/superheroic-scientists/], 25.01.2021

DOI: https://doi.org/10.37189/kwi-blog/20210125-0830 


\section{DuEPublico}

Dieser Text wird über DuEPublico, dem Dokumenten- und Publikationsserver der Universität Duisburg-Essen, zur Verfügung gestellt. Die hier veröffentlichte Version der EPublikation kann von einer eventuell ebenfalls veröffentlichten Verlagsversion abweichen.

DOI: $\quad 10.37189 / \mathrm{kwi}-\mathrm{blog} / 20210125-0830$

URN: urn:nbn:de:hbz:464-20210125-103107-5 